\title{
Treatment of a Neumann boundary condition by a Particle Strength Exchange method.
}

\author{
Rita Benhaddouch \\ Ecole Polytechnique. C.M.A.P. \\ 91128 Palaiseau Cedex \\ rita@cmapx.polytechnique.fr
}

Particle Methods with an integral approximation of the diffusion operator have been successfully developed among others by G.H. Cottet, P. Degond, S. Mas-Gallic and P.A. Raviart for a domain without boundary condition and by $S$. Mas-Gallic with a planar boundary. We extend in this paper the designing of the integral approximation of the diffusion operator that can be used in a straightforward way for a wider class of domain geometries.

In such methods the fluid is represented by a combination of Dirac measures or particles which travel according to the fluid velocity. The problem was then to find an approximation of the second order differential operator in a way that could be treated by a Lagrangian method. This gave rise to the work of Raviart's team $(4,5,6,13)$ where the second order differential operator is approximated by an integral operator. To include the treatment of the diffusion, we allocate each particle a weight which varies according to a first order differential equation in time. In the Particle Strength Exchange Method on $\mathbb{R}^{2}$, the Laplacian operator is approximated by an integral operator in the following way:

$$
\Delta_{\varepsilon} \omega(x)=\frac{1}{\varepsilon^{2}} \int_{\mathbb{R}^{2}} \eta_{\varepsilon}(x-y)(\omega(y)-\omega(x)) d y
$$

where $\eta$ is a symmetric function with a compact support which satisfies the second order moment condition:

$$
\int_{\mathbb{R}^{2}} \eta(z) z_{i}^{2} d z=2, \text { for } i=1,2
$$

and $\eta_{\varepsilon}(x)=\frac{1}{\varepsilon^{2}} \eta\left(\frac{x}{\varepsilon}\right)$.

Since the particle methods are directly connected with quadrature formulae, the approximation of the Laplacian operator by an integral operator is well suited. Moreover, it is possible to include the treatment of a boundary condition by using boundary integral equation. S. Mas-Gallic suggested a method to solve a convection-diffusion equation with a Neumann boundary condition in the half space domain which consists in approximating the Laplacian operator with a two parts integral operator:

$$
\Delta_{\varepsilon} \omega(x)=\frac{1}{\varepsilon^{2}} \int_{\Omega}\left(\eta_{\varepsilon}(x-y)+\eta_{\varepsilon}(x-\tilde{y})\right)(\omega(y)-\omega(x)) d y-2 \int_{\partial \Omega} \zeta_{\varepsilon}(x-y) g(y) d \Gamma(y)
$$

where $\tilde{y}$ is the image point (i.e. the symmetric to $y$ with respect to the boundary), $g$ is the Neumann condition and $\eta$ and $\zeta$ are even compactly supported functions. This work is an extension of the previous works, having always in mind that the goal is to obtain a numerical method using non singular kernels and that the analysis must be made in a way that exhibits properties of the 
kernel(s) that is(are) explicitly solvable.

When solving a convection-diffusion equation, the weight of the particles is locally modified in the vicinity of the boundary in a strip of width $\varepsilon$. The information is then carried along the characteristic curves and diffused within the domain. The convolution integrals are approximated with a quadrature formula where the discretization nodes are the particles. Assuming that $\eta$ and $\zeta$ are related by a compatibility condition, the approximation error is of magnitude $\varepsilon$, provided that $\eta$ fulfills the second order moment condition (2).

In the case where the domain $\Omega$ is the complementary in $\mathbb{R}^{2}$ of a disc of radius $R$, the asymptotic expansion of the operator $\Delta_{\varepsilon}$ contains 2 singular terms of same order that can be canceled in two ways: either by imposing a compatibility condition between the two kernels or by modifying the expression of the approximated diffusion operator. The first option leads to the same type of relationship as the one already introduced by S. Mas-Gallic in the case of the half-space. For more complex geometries (especially non-self homothetic ones) extension of this approach is not straightforward. We suggest the modified diffusion approximated operator:

$$
\Delta_{\varepsilon} \omega(x)=\frac{1}{\varepsilon^{2}} \int_{\Omega} \rho_{\varepsilon}(x, y)[\omega(y)-\omega(x)] d y-\frac{1}{\varepsilon^{2}} \int_{\Omega} \rho_{\varepsilon}(x, y)(y-x) d y \cdot n(P x) g(P x)
$$

where

$$
\rho(x, y)=\eta(x-y)+\eta(x-\tilde{y}) \quad \text { and } \quad \rho_{\varepsilon}(x, y)=\frac{1}{\varepsilon^{2}} \rho\left(\frac{x}{\varepsilon}, \frac{y}{\varepsilon}\right)
$$

$P x$ is the projection of $x$ on the boundary and $n$ is the outward normal to the boundary and $\eta$ is a radial function.

In this formulation, the convolution kernel on the boundary $\partial \Omega$ in (3) is replaced by a convolution kernel within the domain. For any kernel $\eta$ which fulfills the second order moment condition (2), we can formulate an integral approximation of the diffusion operator explicitly.

An outline of the paper is as follows. We give a general presentation of the method. We first prove that the above approximation is of order $\varepsilon$ in $L^{\infty}$ norm. This error estimate shows that, for a given $\varepsilon$, the larger the radius $R$, the better the approximation. Then we prove the convergence of the discretized integral operator on the nodes carried along the characteristic curves under the condition that the space discretization parameter $\mathrm{h}$ is small in comparison with the numerical discretization parameter $\varepsilon$ and that gives the consistency of the particle method. Then using a positivity result established by S. Mas-Gallic and P.A. Raviart, we prove a stability result of the method under the condition that the function $\eta$ is non-negative. Then, combining these results, we obtain the convergence of the method. We first prove that the method converges on the particles, then introducing a smoothing function as it is usual in particle methods we prove the convergence in a more classical sense and that ends the analysis of the semi-discretized scheme.

Finally we give numerical bidimensional illustrations for the computation of a convection-diffusion equation around the cylinder by separating the convective part and the diffusive part.

\section{Presentation of the method.}

Let us introduce some notations and give a rapid statement of the method.

Let's denote by $\bar{D}$ the closed disc of radius $R, \Omega$ the complementary of $\bar{D}$ in $\mathbb{R}^{2}, \partial \Omega$ the boundary of $\Omega$ and by $\left.\Omega_{T}=\Omega \times\right] 0, T\left[\right.$ and $\left.\partial \Omega_{T}=\partial \Omega \times\right] 0, T[$.

We consider in this paper the flow of an incompressible viscous fluid around an obstacle governed by the Navier-Stokes equation in a velocity-vorticity formulation.

ESAIM: Proc., Vol. 7, 1999, $37-45$ 


$$
\left\{\begin{aligned}
\frac{\partial \omega}{\partial t}(x, t)+\operatorname{div}(u \omega)(x, t)-\nu \Delta \omega(x, t) & =0 & & \text { in } \Omega_{T}, \\
\omega(x, 0) & =\omega_{0}(x) & & \text { in } \Omega \\
-\Delta u(x, t) & =\nabla \times \omega(x, t) & & \text { in } \Omega_{T} \\
u(x, t) & =0 & & \text { on } \partial \Omega_{T} \\
\frac{\partial \omega}{\partial n}(y, t) & =g(y, t) & & \text { on } \partial \Omega_{T}
\end{aligned}\right.
$$

where g depends on the velocity $u$ and $n$ is the normal to the boundary outwardly directed to $\Omega$. Let us now mention that this system can be solved by splitting the system into two subsystems where the evolution of the vorticity is calculated once the velocity is given (see the work of G.H. Cottet in the case of a Neumann boundary condition). We shall only consider here the subsystem related to the evolution of the vorticity and thus suppose that the Neumann boundary condition $g$ is given and the velocity is "admissible" in the sense that it satisfies the no slip boundary condition. We denote by $v$ this "admissible" velocity. Suppose $v$ is a sufficiently smooth vector field such that

$$
\operatorname{div} v(x, t)=0 \text {, for }(x, t) \in \Omega_{T}
$$

We will, from now on, concentrate on the following system:

$$
\left\{\begin{aligned}
\frac{\partial \omega}{\partial t}(x, t)+\operatorname{div}(v \omega)(x, t)-\nu \Delta \omega(x, t) & =0 & \text { in } \Omega_{T} \\
\omega(x, 0) & =\omega_{0}(x) & \text { in } \Omega \\
\frac{\partial \omega}{\partial n}(y, t) & =g(y, t) & \text { on } \partial \Omega_{T}
\end{aligned}\right.
$$

Let us denote by $X\left(t, x_{0}, 0\right)$ the characteristic curves which are the integral curves of the vector field $v$ :

$$
\left\{\begin{aligned}
\frac{d X}{d t}(t) & =v(X(t), t) \quad \text { for } t \in] 0, T[ \\
X(0) & =x_{0}
\end{aligned}\right.
$$

As mentioned above, the method consists in the introduction of a small parameter $\varepsilon$ and the replacement of the diffusion operator by an integral operator. Then we introduce a quadrature formula on $\Omega$ in order to define a space discretization. We initially distribute a set of points $\left(x_{k}^{0}\right)_{k \in K}$ in the domain. We define an operator $\Pi_{h}(t)$ which associate to any sufficiently smooth function $f$ a particle distribution a time $t$ by:

$$
\Pi_{h}(t) f(x)=\sum_{k \in K} v_{k}(t) f\left(x_{k}(t)\right) \delta\left(x-x_{k}(t)\right)
$$

where $v_{k}(t)$ and $x_{k}(t)$ denotes respectively the volume and the position of the particle at time $t$ which follows the integral curves of the vector field $v$ starting at the point $x_{k}^{0}$ at time 0 . The 
incompressibility condition (6) implies that the volume of the particles does not evolve in time. The particle method consists in looking for a measure under the form:

$$
\omega_{h}(t)=\sum_{k \in K} v_{k}(t) \omega_{k}^{h}(t) \delta\left(x-x_{k}(t)\right)
$$

where the strengths $\omega_{k}^{h}(t)$ are solution of an ordinary differential equation. Since the velocity is divergence free, the cells of the particles don't dilate in time.

From the definition of $\Delta_{\varepsilon}$ and from the quadrature formula defined by:

$$
\int f(x) d x \simeq \sum_{k \in K} v_{k} f\left(x_{k}\right) \quad \forall f \in C^{o}\left(\mathbb{R}^{2}\right) \cap L^{1}\left(\mathbb{R}^{2}\right),
$$

we derive a discretized version $\Delta_{\varepsilon}^{h}$ of the approximated diffusion operator:

$$
\Delta_{\varepsilon}^{h} \omega_{k}(t)=\frac{\nu}{\varepsilon^{2}} \sum_{i \in K} v_{i}\left[\rho_{\varepsilon}\left(x_{k}(t), x_{i}(t)\right)\left(v_{k} \omega_{i}(t)-v_{k} \omega_{k}(t)-\left(x_{i}(t)-x_{k}(t)\right) \cdot n\left(P x_{k}(t)\right) g\left(P x_{k}(t)\right)\right]\right.
$$

The numerical resolution consists in solving the following system of first order ordinary differential equations

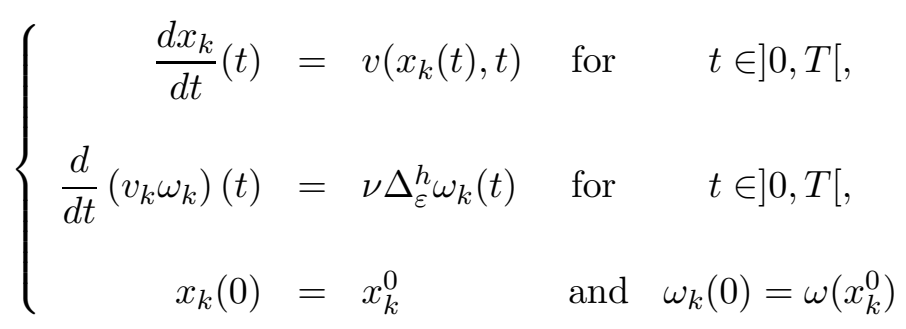

In what follows, for any integer $m \geq 0$ and any $p \in[1,+\infty]$, we denote by $W^{m, p}$ the Sobolev space provided with its classical norm denoted by $\|\cdot\|_{m, p}$ and $W^{-m, p}$ the dual Sobolev space provided with its classical norm denoted by $\|\cdot\|_{-m, p}$.

\section{Analysis of the semi-discretized scheme : Convergence in $L^{\infty}$.}

Proposition 2.1 Assume that $\eta$ is a compactly supported radial function of $W^{1, \infty}\left(\mathbb{R}^{2}\right)$ which satisfies the two second order moment conditions (2).

Then there exists a constant $C>0$ such that, for any function $g$ and for any function $\omega \in$ $L^{\infty}\left(0, T ; W^{3, \infty}(\Omega)\right)$ that satisfy the boundary condition:

$$
\frac{\partial \omega}{\partial n}(x, t)=g(x, t) \quad \forall x \in \partial \Omega
$$

we have

$$
\left.\left\|\Delta_{\varepsilon} \omega-\Delta \omega\right\|_{0, \infty} \leq C\left(\varepsilon+\frac{\varepsilon}{R}\right)\|\omega\|_{3, \infty} \quad \forall t \in\right] 0, T[
$$


Proof:

We introduce a local parameterization in the vicinity of the boundary in a strip of $\varepsilon$ width and we make a local analysis in a disc of radius $\varepsilon$.

We denote by $\Phi_{\gamma}$ the change of variables:

$$
\begin{aligned}
\Phi_{\gamma}: \quad \mathbb{R} \times \mathbb{R}_{+} & \longrightarrow \Omega \\
\left(t_{1}, t_{2}\right) & \longmapsto\left(x_{\gamma}\left(t_{1}\right), y_{\gamma}\left(t_{1}\right)\right)-t_{2} n_{\gamma}\left(t_{1}\right)
\end{aligned}
$$

where $n_{\gamma}\left(t_{1}\right)$ is the unit normal to the boundary outwardly directed to $\Omega$ at the point $\left(x_{\gamma}, y_{\gamma}\right)$. $\left(x_{\gamma}(t), y_{\gamma}(t)\right)$ denotes the couple of Cartesian coordinates relatively to the curvilinear abscissa $t$ of a point belonging to the circle.

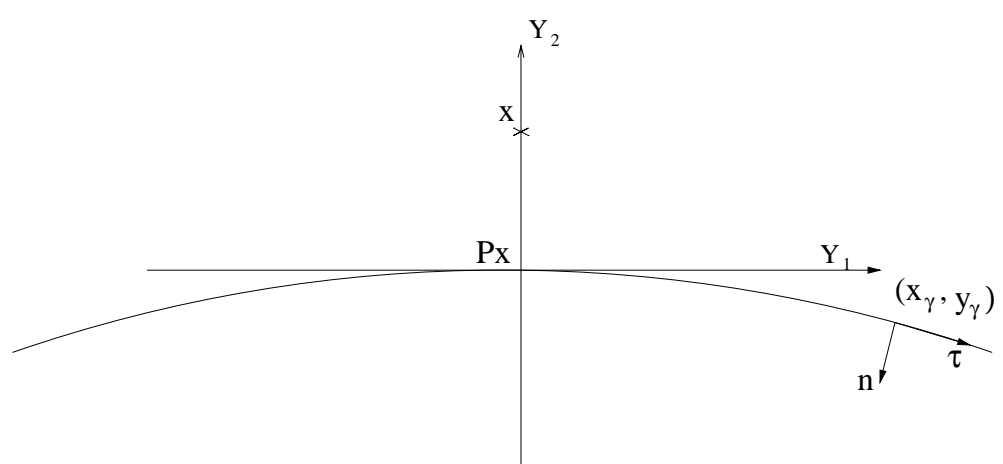

Local parameterization in the vicinity of the boundary.

\section{Remarks:}

1. Beyond a strip of $\varepsilon$ width, we find again the approximated Laplacian in the whole space.

2. For a given $\varepsilon$, the larger $R$ the better the approximation. A direct consequence is that we can extend the analysis made for the case of a cylinder to a more general class of domain shapes where the numerical method will consist in fixing the numerical parameter $\varepsilon$ with respect to the smaller curvature radius.

3. If one wishes to refine the $\varepsilon$ widths strip in order to take into account large fluctuation of the function near the boundary then the distance to the image point can be approximated by the following formula :

$$
|x-\tilde{y}|^{2} \simeq(|x-P x|+|y-P y|)^{2}+|P x-P y|^{2}
$$

This approximated distance may give correct results since when we are very close to the boundary the boundary is almost planar and in the case of a planar boundary the formula is an exact equality.

4. Moreover this approximated distance implies the conservation of the circulation for the case of a homogeneous Neumann boundary condition for the integro-differential model.

$$
\left\{\begin{array}{l}
\frac{\partial \omega}{\partial t}(x, t)+\operatorname{div}(u \omega)(x, t)-\nu \Delta_{\varepsilon} \omega(x, t)=0 \\
\omega(x, 0)=\omega_{0}(x), \frac{\partial \omega}{\partial n}(y, t)=g(y, t)
\end{array}\right.
$$

5. It is possible to derive a conservative form of $\Delta_{\varepsilon}$ 
Let us now define the initial quadrature rule. For this purpose, we introduce two discretization parameters $h_{r}$ and $h_{\theta}$. We denote by $C_{i}$ the circle of radius $r_{i}=R+\left(i+\frac{1}{2}\right) h_{r}$ and $n_{i}=E\left[\frac{2 \pi r_{i}}{h_{\theta}}\right]$ and $\theta_{i}=\frac{2 \pi}{n_{i}}$

where $E[x]$ is the greater integer lower than or equal to $x, \forall x \in \mathbb{R}$.

We set

$$
\Omega_{i}=\left\{\left(x_{1}, x_{2}\right) \in \Omega, r_{i}-\frac{h_{r}}{2} \leq\left|\left(x_{1}, x_{2}\right)\right|<r_{i}+\frac{h_{r}}{2}\right\}
$$

and $v_{i}=h_{r} \theta_{i} r_{i}$. We set $h=\sup _{i}\left\{r_{i} \theta_{i}\right\}$

We now concentrate on the consistency of the numerical discretization of the integral operator. We will here prove the convergence of the discretized operator $\Delta_{\varepsilon}^{h}$ towards the integral operator $\Delta_{\varepsilon}$ when $h_{r}, h$ and $\varepsilon$ go to zero under the condition that the $\varepsilon$ width covers a sufficient number of particles.

For this Let us first prove the intermediate result:

Lemma 2.1 For any integer $m \geq 1$ and any function $f \in W^{m+1,1}(\Omega)$, there exist a positive constant $C$ such that,

$$
\left|\int_{\Omega} f\left(x_{1}, x_{2}\right) d x-\sum_{i=0}^{N} v_{i} \sum_{j=0}^{n i} f\left(r_{i} \cos \left(j \theta_{i}\right), r_{i} \sin \left(j \theta_{i}\right)\right)\right| \leq C\left(h_{r}^{2} C_{R}\left\{f\left\|_{2,1}+C_{R} h^{m}\left(1+h_{r}\right)\right\| f \|_{m+1,1}\right)\right.
$$

where $C_{R}=\sup \left\{1, \frac{1}{R}\right\}$

Proof:

We use the fact that the quadrature rule defined above is of order 2 on a half-infinite interval and of "infinite" order on a closed curve.

Let us now derive from the previous lemma a consistency result.

Proposition 2.2 Assume that $\eta$ is a compactly supported function of $W^{m+1,1}\left(\mathbb{R}^{2}\right)$ where $m \geq 1$, then for all $t \in] 0, T\left[\right.$ there exist two constants $C(u, T)$ and $C^{\prime}(u, T)$, such that for any function $\omega \in L^{\infty}\left(0, T, W^{2, \infty}\right)$ and $g \in L^{\infty}\left(0, T, W^{0, \infty}\right)$, we have

$$
\begin{aligned}
& \sup _{k \in K}\left|\Delta_{\varepsilon} \omega\left(x_{k}(t)\right)-\Pi_{h}(t) \Delta_{\varepsilon} \omega\left(x_{k}(t)\right)\right| \\
& \leq C\left(C_{R} \frac{h_{r}^{2}}{\varepsilon^{3}}+C_{R} \frac{h\left(1+h_{r}\right)}{\varepsilon^{3}}\right)\|\omega\|_{2, \infty}+C^{\prime}\left(C_{R} \frac{h_{r}^{2}}{\varepsilon^{3}}+C_{R} \frac{m}{\left.\frac{h^{m}\left(1+h_{r}\right)}{\varepsilon^{m+2}}\right)\|g\|_{0, \infty}}\right.
\end{aligned}
$$

Proof:

We make the change of variable $(z \longmapsto X(0 ; y, t))$ and apply the previous lemma.

We will now prove the convergence of the method. In this purpose, we will derive from the previous results the convergence of the method in a particle sense, which mean that we will prove that $\omega_{k}(t)$ 
tends to $\omega\left(x_{k}(t), t\right)$ for all $\left.t \in\right] 0, T$. We will next prove the convergence of the particle method in a more classical way. To prove the convergence of the method in a particle sense, we will use a result (the proof of which can be found in (13) ), which is of constant use in particle method.

Lemma 2.2 Given a set $\left(v_{p}^{0}\right)_{p \in K}$, and a function $\left(b_{p}(t)\right)_{p \in K} \in L^{1}\left(0, T ; l^{\infty}(K)\right)$, we consider the following ordinary differential system of equation:

$$
\frac{d v_{p}}{d t}(t)+\sum_{q \in K} a_{p, q} v_{q}(t)=b_{p}(t), \quad v_{p}(0)=v_{p}^{0}
$$

We assume that the coefficients $\left(a_{p, q}\right)$ are such that $\forall p \in K$,

$$
\begin{aligned}
& a_{p, q} \leq 0 \text { for } q \neq p ; \quad \sum_{q \in K} a_{p, q} \geq 0 ; \quad \sum_{q \in K}\left|a_{p, q}\right| \leq C \\
& 0 \leq v_{p}^{0} \leq M, \quad 0 \leq b_{p}(t) \leq B(t) \quad \forall p \in K .
\end{aligned}
$$

Then, problem (13) has a unique solution $\left(v_{p}(t)\right)_{p \in K} \in l^{\infty}(K)$ and this solution is such that

$$
0 \leq v_{p}(t) \leq M+\int_{0}^{t} B(\tau) d \tau \quad \forall p \in K
$$

Let us now focus on the convergence of the scheme on the particles. We will apply the previous lemma following the same idea as S. Mas-Gallic in (11).

Theorem 2.1 Assume that $\eta$ is a compactly supported nonnegative function of $W^{m+1,1}\left(\mathbb{R}^{2}\right)$ where $m \geq 1$, then for all $t \in[0, T]$ there exist two positive constants $C(u, T)$ and $C^{\prime}(u, T)$ depending on $u$ and $T$, such that for any function $\omega \in L^{\infty}\left(0, T, W^{3, \infty}\right)$ and $g \in L^{\infty}\left(0, T, W^{0, \infty}\right)$, we have $\forall t \in] 0, T[$,

$$
\begin{aligned}
& \sup _{k \in K}\left|\omega\left(x_{k}(t)\right)-\omega_{h}^{k}(t)\right| \\
& \leq C \nu\left(\left(\varepsilon+\frac{\varepsilon}{R}\right)+\frac{h_{r}^{2}}{\varepsilon^{3}} C_{R}+C_{R} \frac{h\left(1+h_{r}\right)}{\varepsilon^{3}}\right)\|\omega\|_{L^{\infty}\left(0, T ; W^{2, \infty}(\Omega)\right)} \\
& +C^{\prime} \nu\left(C_{R} \frac{h_{r}^{2}}{\varepsilon^{3}}+C_{R} \frac{h^{m}\left(1+h_{r}\right)}{\varepsilon^{m+2}}\right)\|g\|_{L^{\infty}\left(0, T ; W^{0, \infty}(\partial \Omega)\right)}
\end{aligned}
$$

We will now prove the convergence of the scheme in the more classical sense of functions. As it is of constant use in particle methods, we introduce a smoothing function which allows the transition from a combination of Dirac measures to a regular function. We define an operator $\Pi_{h}^{\varepsilon}(t)$ which is achieved by convoling a particle distribution a time $t$ with a function $\phi_{\varepsilon}$.

$$
\Pi_{h}^{\varepsilon}(t) f(x)=\sum_{k \in K} v_{k} f\left(x_{k}(t)\right) \phi_{\varepsilon}\left(x-x_{k}(t)\right)=\Pi_{h}(t) f * \Phi_{\varepsilon}(x)
$$


where $\Phi_{\varepsilon}(x)=\frac{1}{\varepsilon^{2}} \Phi\left(\frac{x}{\varepsilon}\right)$ is a regular function contracted thanks to the $\varepsilon$ discretization parameter and renormalized in $L^{1}$ norm. We also define a regularized approximated solution $\omega_{h}^{\varepsilon}$ of the particle solution $\omega_{h}$

$$
\omega_{h}^{\varepsilon}(x, t)=\sum_{k \in K} v_{k} \omega_{k}^{h}(t) \phi_{\varepsilon}\left(x-x_{k}(t)\right)
$$

Let us now prove the following intermediate result:

Lemma 2.3 Assume that $\phi$ is a compactly supported function, then for all $f \in W^{2, \infty}\left(\mathbb{R}^{2}\right)$, there exists a constant $C$ such that,

$$
\left\|\left(f-\Pi_{h}(t) f\right) * \phi_{\varepsilon}\right\|_{0, \infty} \leq C C_{R}\left(\frac{h_{r}^{2}}{\varepsilon^{2}}+\frac{h\left(1+h_{r}\right)}{\varepsilon^{2}}\right)\|f\|_{2, \infty}
$$

Proof:

$$
\left\|\left(f-\Pi_{h}(t) f\right) * \Phi_{\varepsilon}\right\|_{0, \infty} \leq \frac{C}{\varepsilon^{2}}\left\|\left(f-\Pi_{h}(t) f\right)\right\|_{0,1}
$$

Next, we get from lemma (2.1) for all $f \in W^{2, \infty}\left(\mathbb{R}^{2}\right)$,

$$
\left\|\left(f-\Pi_{h}(t) f\right)\right\|_{0,1} \leq C\left(h_{r}^{2} C_{R}\|f\|_{2,1}+C_{R} h\left(1+h_{r}\right)\|f\|_{2,1}\right)
$$

which achieve to prove the desired result.

Let us now recall the following lemma the proof of which can be found in (14).

Lemma 2.4 Assume that there exists an integer $k \geq 1$ such that

$$
\left\{\begin{aligned}
\int_{\mathbb{R}^{2}} \phi(x) d x & =1 \\
\int_{\mathbb{R}^{2}} x^{\alpha} \phi(x) d x & =0, \quad \forall\left(\alpha \in \mathbb{N}^{2}\right) \text { where } 1 \leq|\alpha| \leq k-1 \\
\int_{\mathbb{R}^{2}}|x|^{k}|\phi(x)| d x & <+\infty
\end{aligned}\right.
$$

then there exists a constant $C>0$ such that $\forall f \in W^{k, p}\left(\mathbb{R}^{2}\right), 1 \leq p \leq+\infty$

$$
\left\|f * \phi_{\varepsilon}-f\right\|_{0, p} \leq C \varepsilon^{k}\|f\|_{k, p}
$$

Theorem 2.2 Assuming that the hypotheses of theorem (2.1) holds and $\phi$ is a compactly supported function of $W^{k, \infty}\left(\mathbb{R}^{2}\right)$ that satisfies hypotheses (17), then there exists two positive constants $C(u, T)$ and $C^{\prime}(u, T)$ such that $\forall t \in[0, T]$,

$$
\begin{aligned}
& \left\|\left(\omega-\omega_{h}^{\varepsilon}\right)(., t)\right\|_{0, \infty} \\
& \leq C\left(\varepsilon^{k}+C_{R}\left(\frac{h_{r}^{2}}{\varepsilon^{2}}+\frac{h\left(1+h_{r}\right)}{\varepsilon^{2}}\right)+\nu\left(\left(\varepsilon+\frac{\varepsilon}{R}\right)+\frac{h_{r}^{2}}{\varepsilon^{3}} C_{R}+C_{R} \frac{h\left(1+h_{r}\right)}{\varepsilon^{3}}\right)\right)\|\omega\|_{L^{\infty}\left(0, T ; W^{l, \infty}(\Omega)\right)} \\
& +C^{\prime} \nu\left(\frac{h_{r}^{2}}{\varepsilon^{3}} C_{R}+C_{R} m \frac{h^{m}\left(1+h_{r}\right)}{\varepsilon^{m+2}}\right)\|g\|_{L^{\infty}\left(0, T ; W^{0, \infty}(\partial \Omega)\right)}
\end{aligned}
$$


where $l=\max (k, 3)$

Proof:

We deduce the result by splitting the error into three terms, and using the three previous results.

\section{Numerical results.}

The aim of this part is to check the accordance of the theoretical results with some numerical results. To perform a first test of the method, we compare the computed solution to an exact radial solution performed with an explicit regular divergence free velocity such that $\omega=\nabla \times u$. We thus solve the following system of equations.

$$
\left\{\begin{array}{rlrl}
\frac{\partial \omega}{\partial t}+\operatorname{div}(u \omega)-\nu \Delta \omega & =0 & & \text { in } \Omega_{T} \\
\frac{\partial \omega}{\partial n} & =\frac{\partial \vartheta}{\partial n} & & \text { on } \partial \Omega_{T} \\
\omega\left(x, t_{0}\right) & =\omega_{0}(x) & & \text { in } \Omega \\
\vartheta(r, t) & =\frac{1}{4 \pi \nu t} \exp \left(-\frac{r^{2}}{4 \nu t}\right) & & \\
\left(u_{1}, u_{2}\right)(x, y) & =\frac{1}{2 \pi r}\left(\exp \left(-\frac{r_{0}^{2}}{4 \nu t}\right)-\exp \left(-\frac{r^{2}}{4 \nu t}\right)\right)(-y, x) &
\end{array}\right.
$$

As predicted by the theoretical error estimate, for a given initial quadrature rule, there exists an optimal $\varepsilon$.

In the figure (figure 3), we have presented the isovalues at the $100^{\text {th }}$ time.

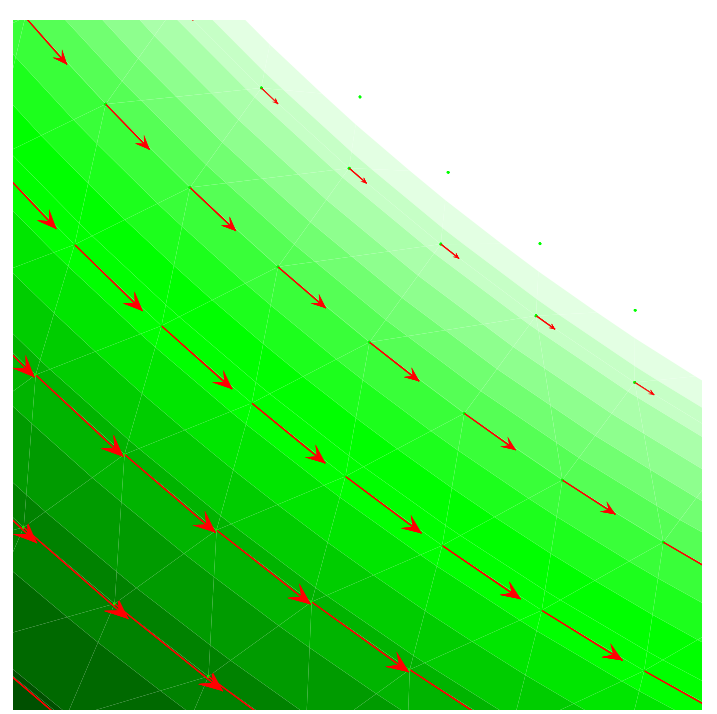

Isovalues at the $100^{\text {th }}$ time step.

For sake of simplicity, we have chosen to perform the previous tests by fixing $h_{\theta}=h_{r}$. Actually, since the quadrature error we introduced is of order 2 on a semi-infinite interval of $\mathbb{R}$ and of infinite order on a closed curve ( $\eta_{1}$ and $\omega$ are infinitely differentiable), one can expect that there is no need in fixing $h_{\theta}$ as fine as $h_{r}$. We performed 3 tests with $h_{\theta 1}=2 h_{\theta}, h_{\theta 2}=2.2 h_{r}$ and $h_{\theta 2}=2.4 h_{\theta}$. 
We observe that $h_{\theta}=2 h_{r}$ leads to a relative error which coincides with the one computed with $h_{\theta}=h_{r}$. But one can not expect in fixing an $h_{\theta}$ much larger since there is also the criteria of optimality in the choice of $\varepsilon$ with regard to $h_{r}$.

\section{Conclusion.}

This numerical method is close to the physical behavior of a slightly viscous fluid since the boundary layer is well discretized thanks to a numerical boundary layer delimited by a numerical viscosity discretization parameter $\varepsilon$. Moreover this numerical method can be applied to thin boundary layers. Some numerical tests performed on a radial solution had showen that using a Gaussian kernel leads to good results for 3 particles per $\varepsilon$ width's strip (the same quality of results requires 13 particles per $\varepsilon$ widths strip with a hat function). This preliminary work had been made for the resolution of the Navier-Stokes equation in velocity-vorticity formulation by a purely Lagrangian method for high Reynolds number flows with a unique lagrangian method. This work is in progress. Let us point out that another Particle Strength Exchange method had been decelopped by researchers in fluid mecanics (9).

\section{References}

1 A.J.CHORIN, Numerical study of slightly viscous flows, J. Fluids Mech., 57 (1973) 785-796.

2 G.H. COTTET, A vorticity creation algorithm for the Navier-Stokes equations in arbitrary domain, in Navier-Stokes equations and related Nonlinear problems, A. Seiquiera ed., Plenium press, New-York, 1995.

3 G.H. COTTET, Boundary conditions and the deterministic vortex methods for the Navier-Stokes equations, in Mathematical Aspects of Vortex Dynamics, R.E. Caflish, ed., Society for industrial and Applied Mathematics, Philadelphia, PA, 1988.

4 G.H. COTTET. And S. MAS-GALLIC, A particle method to solve transport diffusion equation, internal report 115, Centre de Math. Apll.,Ecole Polytechnique.

5 G.H. COTTET. And S. MAS-GALLIC, A particle method to solve the Navier-Stokes system, Numer. Math., 57 (1990), pp 1-23.

6 P. DEGOND AND S. MAS-GALLIC, The weighted particle method for convection-diffusion equation, part I: the case of an isotropic viscosity, part II: the anisotropic case, Math. comp., 53 (1989), pp 485-526.

7 R.A. GINGOLD AND J.J. MONAGHAN, Shock simulation by particle method S.P.H., J. Comput. Phys. 52 (1983), 374-389

8 A. JOLLES, Résolution des équations de Navier-Stokes par des méthodes Particules-maillage, Thèse de doctorat de, Pierre and Marie Curie University, 1989.

9 A. LEONARD, P. KOUMOUTSAKOS AND F. PEPIN, Boundary conditions for viscous Vortex methods., J. Comput. Phys. 113 (1994), 52-61.

10 B. LUCQUIN-DESREUX AND S. MAS-GALLIC, A conservative particle approximation for a boundary value advection-diffusion problem, $M^{2} A N$, Mathematical modeling and numerical analysis, vol 26 $-6-, 1992$.

11 S. MAS-GALLIC, Deterministic Particle Method : Diffusion and boundary conditions, Vortex Dynamics and Vortex methods. C. Anderson and C. Greengard, eds., American Mathematical Society, Lecture in Appl. Mathematics, vol 28 (1992), pp 433-465

12 S. MAS-GALLIC, Particle Approximation of a linear convection-diffusion problem with Neumann boundary conditions, SIAM J. Numer. Anal., VOL 32, No. 4, pp 1098-1125, August 1995.

13 S. MAS-GALLIC AND P.A. RAVIART, Particle approximation of convection-diffusion problems, internal report R 86013, Pierre and Marie Curie University, 1986.

14 P.A. RAVIART, An analysis of particle methods, Numerical Methods in Fluid Dynamics, Lecture notes in Mathematics 1127, Springer Verlag, Berlin, (1985). 